\title{
Libertad de cátedra o socialismo de Estado. El dilema de los estudiantes de la Universidad de Guadalajara en 1933-1937 ${ }^{1}$
}

\author{
Armando Martínez Moya ${ }^{2}$ \\ Universidad de Guadalajara/ISIDM (México) \\ Grupo de investigación HISULA \\ mmarmando@gmail.com
}

Recepción: 20/10/2013

Evaluación: 07/11/2013

Aceptación: 13/12/2013

Artículo de Revisión

DOI: http:/ / dx.doi.org/ 10.9757/Rhela.22 / 09

\section{RESUMEN}

La Universidad de Guadalajara fue arena de un conflicto antagónico escenificado por dos tendencias político-ideológicas que buscaron influir en la orientación de la institución y finalmente en su control: el sector autonómico, defensor de la autonomía universitaria y la libertad de cátedra, y la tendencia socialista, defensora de la reforma de ese signo promulgada por el régimen de la Revolución Mexicana. Esta situación derivó en un movimiento de huelga que de manera intermitente pobló la universidad entre 1933-1937. En este trabajo se abordan algunos aspectos del episodio y su desenlace definitivo. Esta temática ha sido por lo regular tema apologético para la historia oficial de la Universidad, pues el movimiento estudiantil aparece como una efemérides fundacional. En este texto se trata de evidenciar, por medio de relatos que redactaron algunos protagonistas, que la historia rebela otras explicaciones.

Palabras claves: Revista Historia de la Educación Latinoamericana, Universidad, autonomía universitaria, libertad de cátedra, movimiento estudiantil, socialismo, estudiantado, Estado mexicano.

1 Este artículo correspondía a un fragmento del Estado del conocimiento sobre el movimiento estudiantil de Guadalajara 19331937. Por razones de espacio, se redujo este capítulo del el libro colectivo donde si integró Universidad de Guadalajara: más de dos siglos de Historia. Ed. Universidad de Guadalajara. México 2013. Aquí la incluyo por la importancia testimonial de quienes redactaron esos episodios, así como de otras fuentes, de ahí que aborde fuentes documentales directas. Otra versión, tomando otras fuentes de bibliografía y folletería local sobre este tema, la expuse en el Congreso de la Federación internacional de estudios sobre América Latina y el caribe (FIEALC), organizado por la Universidad de Ankara, en Antalya, Turquía del 8 al 11 de octubre de 2013. El texto se articula al proyecto Reformas y movimientos universitarios en Colombia SGI 1295, desarrollado por el grupo Ilustración en América Colonial - ILAC y financiado por la Dirección de Investigaciones de la Universidad Pedagógica y Tecnológica de Colombia.

2 Doctor en Historia y Licenciado en Historia, profesor de la Universidad de Guadalajara, integrante del grupo de investigación Historia y Prospectiva de la Universidad Latinoamericana, adscrito a la Universidad Pedagógica y Tecnológica de Colombia. 
Academic freedom or socialism of state. The dilemma of the students of the Guadalajara University in 1933-1937

\begin{abstract}
Liberdade de cátedra ou socialismo de Estado. O dilema dos estudantes da Universidade de Guadalajara em 1933-1937
\end{abstract}

\section{RESUMO}

The University of Guadalajara was a sand of an antagonistic conflict staged by two political - ideological tendencies that they thought about how to influence in the guidelines of the institution and finally in its control: the autonomic area, defending university autonomy and the academic freedom, and the socialistic tendency, defending the reform of this sign promulgated by the diet of the Mexican Revolution. This situation emerged in a movement that in an intermittent way populated the university between 1933-1937. In this work, there are tackled some aspects of the episode and its definitive ending. This subjectmatter has been as a rule an apologetic topic for the official history of the University, since the student movement appears as one anniversary of its foundation. In this text it is a question of demonstrating, through histories that some protagonists wrote, that the history shows other explanations.

Key words: Journal of the History of American Education University, university autonomy, academic freedom, student movement, socialism, students, Mexican State
A Universidade de Guadalajara foi arena de um conflito antagônico encenado por duas tendências político-ideológicas que buscaram influenciar na orientação da instituição e finalmente em seu controle: o setor autônomo, defensor de autonomia universitária e da liberdade de cátedra, e a tendência socialista, defensora da reforma desse signo promulgada pelo regime da Revolução Mexicana. Esta situação resultou em um movimento que de maneira intermitente povoou a universidade entre 1933-1937. Neste trabalho se abordam alguns aspectos do episódio e seu desenlace definitivo. Essa temática foi, de maneira regular, tema apologético para a história oficial da Universidade, pois o movimento estudantil aparece como uma efeméride fundacional. Neste texto, tratou-se de evidenciar, por meio de relatos escritos por alguns protagonistas, que a história revela outras explicações.

Palavras-chave: Revista História da Educação Latino-americana, Universidade, autonomia universitária, liberdade de cátedra, movimento estudantil, socialismo, alunado, Estado mexicano.

\section{INTRODUCCIÓN}

\section{Una paradoja en la Universidad de Guadalajara}

La Universidad de Guadalajara, la segunda más grande del México, tiene una dilatada historia que se remonta hasta el periodo colonial (1792) y se refunda en 1925. Este último periodo ha generado la consignación historiográfica de una serie de girones históricos que buscan darle sentido a su origen para conquistar su legitimidad, conceptualizando a la institución como producto de las luchas emancipatorios populares que cristalizaron en reapertura, defensa, orientación y destino. Uno de esos mitos es el de su histórico triunfo como proyecto popular ante las asechanzas y amenazas de las fuerzas conservadoras y privatizadoras de la educación. El movimiento estudiantil 
de 1933-1937, escenificado en pleno periodo cardenista (en donde el régimen reivindicaba la educación socialista e incluso implementó una reforma educativa invocándola), se inscribe dentro del conflicto que se escenificaba entonces entre la consecución de un proyecto estatal progresista y quienes reivindicaban la autogestión académica e ideológica de las universidades sin intervención del Estado. En oposición, se configuró un bloque opositor a las políticas del Estado que reivindicaba la libertad y de cátedra y la autonomía universitaria. En este artículo se muestran algunos de los sucesos y circunstancias en las que ocurrió ese proceso, al tratar de dilucidar lo que realmente significó una y otra fuerza, mostrando contradicciones evidentes en los planteamientos tanto de la corriente socialista como la autonomista.

\section{Un escenario convulsionado}

En 1933, el congreso de los diputados de México reformó el artículo tercero Constitucional, al establecer que la educación que impartiría el estado en las escuelas públicas sería socialista como parte del programa político del presidente Cárdenas (1934-1940). Este postulado desató una gran controversia en el país, que derivó en debates y enfrentamientos, incluso violencia y muertes perpetradas por grupos conservadores y fanáticos. La Universidad de Guadalajara no estuvo exenta a esta problemática, en su seno se enfrentaron dos tendencias irreconciliables: en una estaban quienes abogaban por la libertad de cátedra y la autonomía universitaria y postulaba una posición plural y decidir por sí misma sobre sus principios filosóficos e ideológicos. En la otra tendencia estaban los partidarios del régimen político de Cárdenas, quienes defendían los principios de la reforma, considerando que esta generaría la posibilidad de encausar la educación hacia el progreso, la emancipación, la educación popular y una vía pacífica hacia el socialismo. En este trabajo se analizan los principios y acciones de ambas tendencias, así como el derrotero que siguieron, hasta que una de ellas finalmente logró su legitimidad e influencia (la tendencia socialista) y la otra, optó por construir una opción educativa por medio de una universidad particular. Como colofón se comenta la situación en la que ambas tendencias derivaron.

La versión más recurrente respecto al asunto que detonó el movimiento estudiantil en Guadalajara parte de la aplicación de los acuerdos del Congresos de Universidades de 1933 que resolvió orientar la educación superior bajo los lineamientos de la filosofía social del régimen. Sin embargo, 
las propias características locales gestaban ya desde una década antes darle precisamente una orientación de izquierda desde lo educativo. ${ }^{3}$ La fundación de la Universidad de Guadalajara en 1925, será el cultivo más diáfano de esa proyección, pues vino a cristalizar una secular aspiración no solo para darle organicidad y crear un verdadero sistema de educación superior, sino para conquistar su necesaria soberanía al darle a estos estudios un estatus universitario, pues hacía más de medio siglo que el concepto de universidad se había cancelado. ${ }^{4}$ Pero el proyecto de José Guadalupe Zuno, gobernador y concretizador del concepto, estaba además sustentado particularmente en un anhelo popular de que los jóvenes más necesitados llegasen a un nivel profesional, fuese este capacitador-técnico o profesional en la Universidad. Esta postura se inscribía en la convicción de que la tarea reconstructiva de la revolución pasaba por la educación, a fin de ahuyentar el sino de pobreza. La Universidad estaba abierta para todos. ${ }^{5}$ La conversión de estudiante de escuela profesional a alumno universitario implicó un paso inmenso no solamente por lo institucional -ser universitario-, sino para que ese imaginario fuese configurando una comunidad de estudiantes y profesores que empezaran a construir una identidad de pertenencia desde ese halo universitario. Así que poco había que reclamar en ese momento por parte del estudiantado debido a las bondades de un decreto que expandía y articulaba la formación profesional en su majestad universitaria desde 1925.

El proyecto de Zuno tenía una clara justificación ideológica que lo haría, por lo tanto, entrar en tensión unos años después. Basta revisar el discurso de apertura de Enrique Díaz de León, ${ }^{6}$ primer rector de la universidad, para constatar su signo político, que si bien partía de los postulados nacionalistas y populares de la revolución mexicana -principios que la reconocían como laica, progresista y vinculada a las "clases populares-, estos postulados debían imponerse, no consensarse. Tenía confianza este grupo en una sociedad tapatía, que parecía ver a la universidad como la concesión material de un anhelo revolucionario ubicada precisamente en el corazón del

3 Alma, Dorantes. El conflicto universitario en Guadalajara 1933-1937, (Guadalajara: INAH/SC 1993), 133.

4 Desde el último tercio del siglo XIX y hasta 1925, la Escuelas de educación Superior funcionaron dependientes directamente del gobierno del Estado de Jalisco. Cfr: Marina Mantilla Trolle y Armando Martínez Moya. “Jalisco a la hora de su Universidad”. en Universidad de Guadalajara: más de dos siglos de Historia, (Guadalajara: Universidad de Guadalajara, 2013), 183-236.

5 Ver a este respecto sus reflexiones en "Reminiscencias de una vida". (Guadalajara: Universidad de Guadalajara, 2011).

6 Alfredo Mendoza Cornejo, Antología del pensamiento de Enrique Díaz de León. Colección: Universitarios Ilustres. Vol. III. (Guadalajara: Universidad de Guadalajara, 1988)También Enrique Díaz de León, "Discurso de inauguración del Congreso de Universitarios Mexicanos", en Cuadernos Universitarios (Guadalajara, 1948), 31. El perfil de sus preocupaciones políticas e ideológicas son un claro concepto reivindicador para la obra de la revolución mexicana, la cual debe cristalizar con la obra social, puede verse también por medio de todos sus discursos y escritos. 
occidente mexicano. Lo que importaba era que hubiera ya universidad. Su existencia imponía, daba peso, pero en lo que respecta a su modelo operacional no había entonces mucha comprensión. Se suponía que representaba un modelo democrático. No fue así, porque el mismo sistema político, si bien estaba envuelto en un proyecto popular, era impulsado desde la cúspide, hay pues un mimetismo entre el nuevo régimen revolucionario y la naciente universidad.

De todos modos, no existió ninguna oposición o crítica en los momentos de la fundación universitaria. Se percibe una especie de unanimidad emotiva que ve en ese y otras acciones (inicio de la reforma agraria, la apertura de presas hidráulicas y créditos agrícolas, la escuela rural mexicana para niños campesinos, la expropiación petrolera, el muralismo y la organización obrerosindical), la cristalización de los logros de la revolución. Al hojear la prensa de la época, parece existir una suerte de optimismo reconfortante. ${ }^{7}$ Pocos pensarían que apenas ocho años después la Universidad se vería sacudida por la pugna entre ese sector nacionalista popular-estatista, vinculado fuertemente a la filosofía de los gobiernos revolucionarios de esos años (Obregón, Calles, Cárdenas), y los sectores refractarios que, amparándose en la coyuntura de la libertad de cátedra y la autonomía, se opusieron frontalmente a la educación "socialista". El debate y triunfo en la Universidad de México de la libertad de cátedra y el autonomismo animó y envalentonó a quienes en Guadalajara no aceptaban una universidad doctrinaria, por más que se asumiera como producto de la revolución.

Tal vez, el desfase entre los antecedentes intelectuales en los contextos de la universidad de México y de Guadalajara ayude a explicar la transposición de una pugna que en Jalisco derivó hacia un agudo conflicto. La Universidad de México tenía ya el antecedente de su refundación en 1910, bajo la egida de la filosofía trascendentalista y universal que le imprimió Justo Sierra en su discurso inaugural, muy distante de las palpitaciones reivindicativas y populares de Enrique Díaz de León (primer rector) y su generación a partir de 1925, en donde se veía su comunión con el reformismo del Estado. En el discurso de Sierra ${ }^{8}$ se recurre a la diversidad espiritual como culmen del saber académico, concepción que tiene su raíz en el debate humanístico que intenta romper con el penetrante positivismo y mecanicismo. El núcleo y

\footnotetext{
7 Ver los periódicos El Informador y El Jalisciense (1924-1925). Las reseñas en torno a su futuro establecimiento y cuando es finalmente fundada son apologéticas y de reconocimiento al régimen estatal.

8 Javier García Diego, "El proyecto universitario de Justo Sierra: circunstancias y limitaciones", en Cultura y política en el México Posrevolucionario (México: INEHRM, 2006), 23-40.
} 
antecedente de esta reacción es El Ateneo de la juventud, tesis que recogió con gran talento Antonio Caso, heredero de esa estela de pensadores que en la ciudad de México se había forjado en las tertulias, en la Escuela Nacional de Jurisprudencia y en la Nacional Preparatoria. ${ }^{9}$

En cambio, en Guadalajara, para J. Guadalupe Zuno, Díaz de León y su grupo generacional lo sustancial es el vínculo del saber con el trabajo, con la justicia social, porque reivindica al ser humano. Es sin duda un planteamiento marxista clásico que busca en la humanización de la actividad humana el destierro del trabajo explotado. Este precepto se amalgama con la reivindicación de la gesta revolucionaria. En México, este frente está representado por Lombardo Toledano. ${ }^{10}$ Pero esta doctrina debía ser impuesta, no consensada, tal como lo era en la Unión Soviética después de la muerte de Lenin, aprovechando aquí en México la coyuntura cardenista-. La advertencia era no caer en el garlito de la confrontación con otras ideologías (la libertad de cátedra) porque podía distraer la oportunidad de implementar los postulados más avanzados. El debate plural encierra -para esta tendencia-, un peligro por los antecedentes dogmáticos del conservadurismo que podían distraer y enajenar a un estudiantado que debía orientarse aceleradamente a contribuir a acabar con la justicia social una vez que fuese profesionista. ${ }^{11}$

Estas dos tesis -la libertad entendida como debate plural y la estrategia de una confluencia trascendental (por "mi raza hablará el espíritu") y la libertad concebida como liberación material de la sociedad ("piensa y trabaja")explican la diferencia conceptual e histórica de esas intelectualidades que se nuclearán durante las últimas dos décadas en ambas ciudades. He ahí uno de los orígenes del conflicto. En la universidad nacional lo que está atrás es el pensamiento de una mentalidad liberal, que se nutre de los planteamientos universalistas. En la contraparte, se trata de una generación de hombres comprometidos con las condiciones sociales imperantes en busca de una oportunidad, su mente está impregnada de un mesianismo reivindicativo. Para estos, la clave está en no desperdiciar la coyuntura histórica. Esta

\footnotetext{
9 Cfr: Ma. del Carmen Rovira, "El Ateneo de la juventud”, en Una aproximación a la historia de las ideas filosóficas en México. Siglo XIX y principios del XX, (México: UNAM, 1997).

10 La influencia de Lombardo Toledano con la generación de Díaz de León no fue solo doctrinaria sino personal. El encuentro entre todos ellos fue directo desde el primer congreso de universidades en 1933. Toledano visitó la Universidad de Guadalajara ese mismo año, donde expuso directamente sus ideas. Alfredo Mendoza Cornejo, Enrique Díaz de León. Político, ideólogo y pedagogo de la Universidad de Guadalajara. (Guadalajara: Universidad de Guadalajara, 1992).

11 Ver las argumentaciones de Lombardo Toledano, en su polémica con Caso. Rumbo a la Universidad. Testimonio de la polémica Antonio Caso/Lombardo Toledano. Colección Metro. No 1 (1973).
} 
concepción se fue fraguando desde el Centro Bohemio de José Guadalupe Zuno, que nucleó a diversos pensadores y artistas en busca de una identidad cultural. Ahí ocurre la raíz de la génesis educativa y espiritual.

\section{Cómo empezó todo}

El acontecimiento que detonó el deslinde sobre el papel de las universidades y su orientación fue el Primer Congreso de Universitarios mexicanos, celebrado del 7 al 14 de septiembre de 1933 en la Escuela Nacional Preparatoria en la ciudad de México. La Delegación jalisciense, orientada por la reforma:

[...] exacerbó la confrontación entre la tendencia liberal representada por la Universidad Nacional Autónoma de México y la "socialista" defendida por la Universidad de Guadalajara y otras universidades de provincia. [...] Ya en la práctica política, la iniciativa en el choque político que se desarrollaba, la reclamó para sí la rectoría de la Universidad de Guadalajara, la cual sin más ni más, echó a andar una campaña de "depuración" del magisterio en cumplimiento del Primer Congreso de Universitarios. A mediados de octubre de 1933 los primeros sacrificados fueron los profesores Efraín González Luna, Emilio Robles de León y Andrés B. Núñez, quienes participaban en el grupo de intelectuales que se oponían a la reforma socialista. ${ }^{12} \mathrm{El}$ cese de este grupo de profesores solo fue el principio. Luego se inició una purga en cascada que transcurrió fulminante y que llegó hasta los conserjes de escuela durante el resto del año. Por su parte, los enemigos de la reforma, apoyados cada vez más abiertamente por el clero católico, utilizaban el descontento creado por las medidas represivas aplicadas en su prejuicio. [...] La gran masa del magisterio universitario no estaba siquiera enterada de la significación y alcance de la reforma y por lo tanto no tenía en esos momentos una posición definida, lo cual contribuyó a incrementar la oposición a la reforma socialista. ${ }^{13}$

La Universidad de Guadalajara había surgido como una institución patrimonialista, es decir, bajo la egida de un jefe político, José Guadalupe Zuno, su más importante personero fue el primer rector, Enrique Díaz de León, que a su vez se convirtió en el líder del grupo que copó el consejo

12 El Informador, 18 de octubre de 1933; El Universal, 22 de octubre de 1933

13 Laura Patricia Romero, Coordinadora, "Jalisco desde la Revolución. Tomo 5: Movimientos Sociales. 1929-1940", en Rivera (Gobierno del Estado de Jalisco: Universidad de Guadalajara, 1988), 279. 
universitario y los principales puestos directivos. Su bandera reivindicaba la educación popular, nacionalista, capacitadora. Sin embargo, gran parte de los alumnos que ingresaron a partir de su fundación no pertenecían precisamente al proletariado, sino a clases medias y de mayor posición social, pues no obstante que las cuotas escolares eran bajas, no cualquiera podría sostener una carrera superior. Esta gran franja de alumnos fuer la que de inmediato se opuso a plegarse al adoctrinamiento socialista:

El 23 de octubre de 1933 se iniciaron los brotes de protesta en la Escuela Preparatoria de Jalisco y en la Escuela Normal. Los estudiantes liberales y católicos, aliados con los comunistas, organizaron de inmediato el comité Pro-laicismo, integrado por Agustín Pineda, Dionisio Fernández, Jorge Garabito y Roberto Pardo, organismo que el mismo día 23 difundió un manifiesto en el que se argumentaba entre otras cosas el carácter anticonstitucional y la improcedencia de la reforma socialista dentro de un régimen capitalista como era el mexicano. El documento expresaba también su solidaridad con el movimiento de la UNAM y solicitaba la renuncia del rector Enrique Díaz de León y el desconocimiento de la FEJ. Ese mismo día estalló la huelga en la Escuela Preparatoria de Jalisco. ${ }^{14}$

La huelga detonó además una serie de problemáticas que la institución iba padeciendo debido seguramente al modelo vertical que fue establecido desde un principio, caracterizado por el autoritarismo y ausentismo de los catedráticos, la falta de apoyos para el estudio, la intransigencia de las autoridades para atender problemas escolares cotidianos. ${ }^{15}$ Pero en realidad, lo que incitó a la huelga fue la cuestión ideológica, pues las posiciones entre los dos bandos no parecían encontrar mediación.

Los huelguistas llamaron a la generalización del movimiento y denunciaron también un sinnúmero de irregularidades que padecía la Universidad. De hecho, pues en esos momentos ya funcionaba un amplio frente anti-reformista integrado por liberales, comunistas [y] católicos. ${ }^{16}[\ldots]$ Luego de la distribución del manifiesto mencionado, los estudiantes huelguistas se fueron al centro de la ciudad de Guadalajara gritando sus principales demandas: libertad de cátedra, autonomía universitaria, participación paritaria de los estudiantes en el Consejo Universitario,

14 Mario Rivera Ortiz, El estudiantado una nueva clase social. s/e. (México: Guadalajara, 2007), 47.

15 La apología historiográfica ha evitado revisar fuentes como la de los libros de registro de incidencias. Allí es posible observar el sinnúmero de reclamos, peticiones y quejas del estudiantado. Las constantes altas y bajas del profesorado y otros problemas escolares cotidianos. Archivo Histórico de la Universidad de Guadalajara. Libros 1, 2 y 3. 1925-1933.

16 Rivera, "Título del artículo", 47-48. 
desconocimiento de la FEJ, creación de la casa del estudiante pobre, disminución del costo de la matrícula. Solidaridad con los estudiantes de la UNAM y con el ala izquierda del estudiantado cubano y la destitución del rector Enrique Díaz de León. ${ }^{17}$

Es interesante observar que los estudiantes afiliados al partido comunista no se adhirieron al bloque socialista sino al autonomista, al seguir los lineamientos de su comité central que no apoyó inicialmente el programa político que levantaba el general Lázaro Cárdenas, quien era el candidato oficial y seguro "triunfador" de los comicios federales. Las acciones vertiginosas de los huelguistas permitieron que encabezara el movimiento y el mayor número de alumnos los apoyaran, en detrimento de los socialistas quienes, aunque tenían el apoyo de las autoridades, no pudieron evitar la pérdida del inmueble central y de las escuelas.

Aquí es necesario acotar que los universitarios comunistas ${ }^{18}$ dirigidos por Lidio Rodríguez y Agustín Madrueño, ya expulsados de la Universidad, continuaron luchando por la reducción de las colegiaturas, la participación de los estudiantes en la dirección de la Universidad y por el cese del rector "penerreano"(del Partido Nacional Revolucionario) Enrique Díaz de León."19 [...]"La manifestación de día 23 de octubre culminó en el edificio central de la Universidad en donde hubo un breve diálogo sin acuerdo alguno, al cabo del cual fueron expulsadas las autoridades universitarias y ocupado el edificio por los huelguistas. A continuación se integró formalmente un comité general de Huelga en el que participaron Enrique Pardo Pulido, Jorge Gómez Collazo, Rodolfo Pérez Plascencia, Alfredo García y otros. Se integró también un Comité Ejecutivo de Huelga, cuyo secretario General fue Enrique Pardo. ${ }^{20}$

La reacción del grupo socialista se hizo sentir con el apoyo del Estado, es aquí donde se hacen muy singulares las características del movimiento, pues el grupo progresista, que en otras latitudes regularmente enfrentaría al Estado, en este caso es apoyado por él, incluso por el ejército:

\footnotetext{
17 Rivera, "Título del artículo", 48.

18 Los estudiantes comunistas están agrupados nacionalmente en la Federación de Estudiantes Revolucionarios, cuyas políticas y estrategias las decidían en asambleas nacionales. De esta agrupación es de donde se determinó que los comunistas de Jalisco se adhirieran a la corriente autonómica y no a la socialista.

19 “Periódico", El Machete, 30, octubre, 1933, en: Rivera. óp. cit., 48.

20 El Informador, 24, Octubre, 1933, en Rivera, el estudiantado, óp. cit., 48.
} 
Por su parte, el Consejo General Universitario reunido en la Facultad de Medicina el 24 de octubre, dio todo su apoyo al rector Díaz de León y designó al gobernador Allende como mediador del conflicto. [...] Paralelamente la FEJ convocó a una asamblea extraordinaria en la que aparentemente obtuvo el voto solidario de la mayoría de las sociedades de alumnos de la Universidad y ya con este respaldo actuó de consuno con la rectoría y se enfrentó al Comité de Huelga publicando un amplio manifiesto en cuyo texto se afirmaba que el paro era algo ajeno a la "clase estudiantil" y obedecía a fines "personalistas". ${ }^{21}$ Inmediatamente después de dicho posicionamiento de la dirección de la FEJ presidida por José Parres Arias, fue sustituida por un grupo de estudiantes huelguistas encabezados por Carlos Cuesta Gallardo." 22 [...] "El 25 de octubre los huelguistas fueron desalojados de la rectoría por el ejército y la policía, siendo esta la primera vez que en el Estado de Jalisco se empleaban las fuerzas armadas para reprimir al estudiantado. ${ }^{23} \mathrm{Al}$ dia siguiente los paristas llevaron a cabo un mitin en el jardin "Agustin Rivera" ubicado frente a la Escuela Preparatoria de Jalisco, escuela que nuevamente fue tomada por los huelguistas. El gobernador del Estado Sebastián Allende, volvió a solicitar la intervención de la tropa para evacuar a los ocupantes. Desalojados de la escuela preparatoria los huelguistas se fueron en manifestación hasta Palacio de Gobierno donde una comisión estudiantil entró a dialogar con el gobernador, mientras Rodolfo Pérez Plascencia, Gabriel Gutiérrez García y J. Jesús Madrueño hacían uso de la palabra en la plaza de armas. De tal diálogo nada resultó y los estudiantes prosiguieron su manifestación hacía el edificio central de la universidad, mismo que fue reocupado por los manifestantes después de desarmar a la guardia de agentes policiacos y destrozar puertas y muebles de oficina. El ejército intervino por tercera vez para recuperar el inmueble y en esa ocasión detuvo a 155 paristas que fueron conducidos a la Penitenciaría del Estado. Para justificar su politica progresiva el gobernador del Estado había dicho a los estudiantes con toda solemnidad: el principio de autoridad está sobre todos los intereses y ustedes lo han olvidado. ${ }^{24}$

Hace falta analizar las incidencias y consecuencias de lo que pasó en cada escuela y lo que implicó la participación de la sociedad tapatía. Hay sin duda una ausencia de fuentes documentales para saber qué fue lo que

21 El Informador, 25, Octubre, 1933, en Rivera, el estudiantado, óp. cit., 49.

22 Rivera, el estudiantado, óp. cit., 48-49.

23 El autor se ha equivocado, tal vez por no conocer la historia de los movimientos estudiantiles en la ciudad antes de la fundación de la Universidad de Guadalajara.

24 El Jalisciense, 27, octubre, 1933, en Rivera, óp. cit., 49. 
sucedió en cada escuela, en cada salón de clases y cómo se fue bifurcando el estudiantado. Por lo agitado de los acontecimientos, las autoridades no registraron puntualmente las incidencias cotidianas, los acontecimientos los envolvieron, por ello no hay registros. Se han hecho necesarias las bitácoras de la memoria que los mismos alumnos registraron por su cuenta. ${ }^{25}$ Lo que es cierto es que en todas las escuelas había una agitación fervorosa. Las dos tendencias se peleaban palmo a palmo a sus compañeros.

Para estos momentos ya se habian sumado al movimiento de huelga los alumnos de la Escuela de Jurisprudencia, Normal para señoritas, Comercio e Ingeniería y el día 27 un contingente de estudiantes de Medicina se congregaron en el jardín botánico y desfiló por las calles de la ciudad demandando la libertad de sus compañeros encarcelados y la renuncia del rector Díaz de León." ${ }^{26}[. .$.$] "Ese mismo día Enrique$ Díaz de León, quien poco antes había declarado a la prensa que el movimiento era "intrascendente y minoritario", con peticiones inconexas y contradictorias dirigido por una mezcolanza de comunistas y elementos de filiación clerical [...] pequeño grupo de amotinados", ${ }^{27}$ tuvo que presentar su renuncia irrevocable y abandonar la Universidad de Guadalajara por tercera y última vez. ${ }^{28}$

La capacidad de organización y su amplia cobertura ganada en poco tiempo parecía dar a los autonomistas la virtud de ir siempre adelante y ganar desde un principio el liderazgo estudiantil y con ello la aceptación de la mayoría del estudiantado. Pero esta capacidad no era gratuita. Atrás había toda una red de apoyo que estaba ya fogueada por su enfrentamiento con el Estado mexicano en la cristiada. ${ }^{29}$ Era el conservadurismo tapatío, cuya belicosidad y radicalidad se mostró más fehacientemente desde 1928 en la región. "Es una derecha radical, fundamentada en un nacionalismo a ultranza que se caracterizó por su negativa a aceptar las reformas que implementó el régimen posrevolucionario." ${ }^{30}$ Jalisco es un hervidero de la Derecha. Surgen agrupaciones que buscan detener por cualquier medio las reformas cardenistas. ${ }^{31}$

25 Ver al respecto: Armando Martínez Moya, "Voces de huelga desde el corredor" en Universidad de Guadalajara: más de dos siglos de Historia, (Guadalajara: Universidad de Guadalajara, 2013), 237-284.

26 El jalisciense, 28, octubre, 1933, en: Rivera, el estudiantado, óp. cit., 49.

27 Estas expresiones fueron muy recurrentes en la prensa, sin explicitar ni justificar estos calificativos

28 Rivera, el estudiantado, op. cit., 49.

29 Hugh Gerard Campbell, La derecha radical en México, 1929-1940. México: Colección Sepsetentas, septiembre, 1976.

30 Patricia Valles, "La derecha jalisciense en el periodo de la consolidación del Estado mexicano (1929-1940)", en Miscelánea Jalisciense, (Guadalajara, El Colegio de Jalisco. 1999)

31 Aparece la Cofradía de los Caballeros de Cristo Rey, así como las organizaciones paramilitares "Las legiones", y la "Asociación Revolucionaria Mexicanista." Que tenían como grupo de choque "las camisas doradas". En esos años se va a fundar la Unión Nacional Sinarquista, grupo de tendencia fascista. Valles, "La derecha jalisciense en el periodo de la consolidación...", 21, 24, 29. Sin embargo, durante dos años la caracterización del gobierno fue considerada fascista por la forma como cooptaba 
Todo ese conglomerado de organizaciones políticas jaliscienses estaban orientadas desde esta tendencia, muchas de las cuales se habían fraguado desde el gobierno católico de López Portillo y Rojas en 1912 y consolidado en la lucha cristera, lo que dio lugar también como respuesta a la multiplicación de organizaciones de izquierdas, muchas de ellas antirreligiosas, sin duda porque hacia este frente había derivado el enfrentamiento más crudo, ${ }^{32}$ pero también por la influencia callista. ${ }^{33}$ Tal vez el partido comunista era la única agrupación que trataba de explicarse la situación desde una posición más estructural, mas contextualizada. ${ }^{34}$

Pocos días después, el conflicto universitario asumía grandes proporciones y violencia, amenazando con generalizarse el resto de la república. El autoritarismo estatal exacerbado la efervescencia estudiantil que, según se dijo, hacía peligrar la estabilidad social en su totalidad. El gobierno de Sebastián Allende recurrió a la represión administrativa, policiaca y militar, pero no logró tranquilizar la universidad. Por su parte, el gobierno federal, reiteradamente había rehusado intervenir y no estaba en condiciones de echar mano de formas represivas mayores porque ya se había abusado de ellas previamente y el país vivía una aguda crisis politica, de manera que, en tales condiciones, Allende opto por una salida semilegal que funcionó como una especie de armisticio temporal. Y esa medida fue precisamente la clausura de la Universidad de Guadalajara, aprobada con premura por el congreso local el 28 de octubre de 1933. Allende expidió el decreto No. 3857 y fue autorizado por el Congreso para que, cuando lo estimara conveniente, organizara la educación superior socialista y expidiera la ley orgánica y el reglamento correspondiente. Inmediatamente fueron cerradas las escuelas y facultades de la Universidad excepto la Escuela Politécnica y la Práctica anexa a la Normal." $" 35$

"Ante el cierre de la universidad, el comité de Huelga y otros organismos se auto disolvieron para dar paso a una nueva generación de agrupamientos estudiantiles. [...] "La campaña de propaganda que hacían

corporativamente a las masas.

32 Sin embargo, la disputa por la educación no arrió esa bandera, incuso por encima de las problemáticas curriculares o institucionales el asunto religioso fue clave.

33 Para Alma Dorantes existe una diferencia importante "entre el socialista de los callistas y el impulsado por los cardenistas de Cárdenas. Los primeros enfatizaron la dimensión anticlerical y el repudio a la religión.” Dorantes, "El conflicto universitario en Guadalajara 1933-1937”, 118. La cristiada es la evidencia más clara. Sin embargo, ya en el gobierno cardenista, la tendencia antirreligiosa continuó.

34 El Machete. Órgano del Partido comunista de México, 1933-1937. Corresponsalía de Jalisco.

35 El Jalisciense. 29, octubre, 1933, en: Rivera, el estudiantado, óp. cit., 50. 
el Comité Pro reforma Universitaria, el FREJ, la rectoría y todos los organismos burocráticos del gobierno acusando a la oposición estudiantil de "reaccionaria" y "contrarrevolucionaria" no lograba convencer a la mayoría de los universitarios, ya que en realidad, la huelga revindicaba demandas democráticas y de su lado estaban instituciones de la sociedad civil y politicas de izquierda y de derecha como eran la Iglesia Católica, la UNAM, la Liga de Escritores y Artistas Revolucionarios (LEAR), el Partido Comunista Mexicano y las secciones sindicales del Sindicato de Trabajadores Ferrocarrileros de la República Mexicana. En esta confrontación tales formaciones llegaron a desmentir públicamente que la huelga estudiantil estuviera inspirada por enemigos de la Revolución mexicana - La LEAR incluso, llega a acusar a la FEJ y al rector de ser "marxistas" $y$ "comunistas incapaces". ${ }^{36}$

"Inmediatamente después de la clausura de la Universidad de Guadalajara el CPRDE presentó un pliego petitorio al gobernador del Estado, con demandas muy semejantes a las contenidas en el manifiesto del 23 de octubre. Destacaba en dicho texto su preocupación por la supuesta intervención de fuerzas públicas "extrañas" en el conflicto y clamaban por una universidad "pura". Paralelamente el comité realizaba, con la ayuda de los dirigentes de la CNE, actos de masas en las plazas de la ciudad de Guadalajara, en los que acusaban al gobierno del Estado de tratar de impedir el progreso cultural de Jalisco y se ufanaban de [sic] que sus banderas ya ondeaban en las ciudades de México, Durango y Saltillo. Ante la cerrazón del gobierno estatal el CPRDE viajó a la ciudad de México para entrevistarse con el presidente de la República y pedirle la reapertura de la universidad y su autonomía, pero el general Abelardo Rodríguez se negó a recibirlos y a intervenir en el conflicto aduciendo que se trataba de problemas que deberían ser resueltos por el gobierno local con plena soberanía federal. ${ }^{37}$

El FREJ formuló un proyecto de Ley Reglamentaria de la Cultura Superior para ser entregada al congreso estatal y en el mes de noviembre de 1933 propuso la formación del Centro de Estudios para Estudiantes, Obreros y Campesinos "con el fin de lograr la unión de esas 3 clases sociales". El FREJ también proponía la formación de escuelas "libres", con el fin de dar continuidad a los cursos interrumpidos por la clausura de la universidad. [...] El día 15 (de Noviembre) se rompieron entre el

36 Alfredo Mendoza Cornejo, Organizaciones y Movimientos Estudiantiles en Jalisco de 1900 A 1937 , en Rivera. El estudiantado óp. cit., 51.

37 Periódico Excélsior, 2, Noviembre, 1933, en: Rivera, el estudiantado. óp. cit., 52. 
CPRDE y el gobierno estatal, ante la negativa de conceder la autonomía y la libertad de cátedra para la universidad. El comité respondió desintegrándose para formar el Directorio de Huelga (DH) que de inmediato desplegó un raid de mítines relámpago en los jardines del Santuario, Botánico, San Francisco, Plaza de Armas y Plazuela de la Universidad, que culminaron en la toma de la toma de la Escuela Normal, el cierre de sus puertas y la colocación de la bandera rojinegra. ${ }^{38}$

La cobertura nacional ayudó a impulsar al movimiento prosocialista. El periódico El Jalisciense, que era el de mayor circulación local, se inclinaba a este grupo. La reunión en diciembre de 1933 en la ciudad de México del Congreso del Partido Nacional Revolucionario refrenda el plan sexenal ${ }^{39}$ al impulsar el programa de gobierno de Cárdenas. Esta situación les dio confianza y aceleró las acciones de los socialistas, pues veían en el horizonte político que en los años siguientes la política educativa coincidía con sus propósitos políticos. Como lo fue a nivel nacional, el enemigo identificado fue la iglesia y su propaganda fanatizante, así como las fuerzas conservadoras y derechistas. Es curioso que esta tendencia, que se preciaba de fundamentarse en el marxismo, no orientara sus baterías al sistema capitalista, pues bastaban las reivindicaciones laborales para sentirse identificado con el socialismo del Estado. Según Laura Patricia Romero, esta orientación doctrinaria obedecía a la influencia política del callismo que se sustentaba en el jacobinismo francés y la masonería. ${ }^{40}$ Estos grupos, neófitos, aún de una formación sólida doctrinaria, se dejaban influenciar por las consignas y ataques que se les promovía desde el gobierno para quien el empresariado industrial y rural debía ceder ante las conquistas sociales pero no desaparecer.

La Universidad de Guadalajara reflejó desde un principio la heterogeneidad ideológica del estudiantado. Asistían a sus aulas, estudiantes por lo regular con cierto grado de politización pues los años veinte previos a sus estudios vivieron bajo ese caldo de cultivo que fue la revolución mexicana y después la lucha cristera. Dos fenómenos con el mismo tipo de protagonistas: el campesinado, pero con signo político radicalmente diferente. Politización que no era otra cosa que una guerra de consignas, acusaciones, versiones e invenciones de lo que era la situación política en Guadalajara. Había comunistas

\footnotetext{
38 Rivera, el estudiantado, op. cit., 52.

39 Gilberto Guevara Niebla, Las luchas estudiantiles en México. Tomo II. (México: UAG/AUZ, 1986), 60-61.

40 Laura Patricia Romero, "Los estudiantes entre el socialismo y el neoconservadurismo", en Jalisco. Desde la Revolución. Movimiento Social. Tomo V, (Guadalajara: Gob. del Estado de Jalisco, UdG, 1988), 266.
} 
y anticomunistas, afiliados al PNR. "En sus aulas convivían sin fricciones estudiantes liberales, católicos y jacobinos". ${ }^{41}$ Hasta que estalló la huelga, cada quien se ubicó en su frente.

Ante la presión estudiantil y el peligro de expansión del movimiento a otros puntos del país, Sebastián Allende hizo pública una nueva propuesta para solucionar el conflicto, en la que aceptaba la obligación que tenía el Estado para impartir educación superior y la libertad de cátedra, siempre que no se acatase a las instituciones de la revolución. Así mismo sugirió la integración de un consejo universitario en el que se admitiría la presencia estudiantil, más no en proporción paritaria. [...] $Y$ en este momento crucial fue precisamente en el que el general Plutarco Elías Calles, "el jefe máximo" de la revolución, intervino abiertamente haciendo a un lado de manera brutal la jerarquía oficial. Esta vez Calles intervino para apoyar al Gobernador Allende y "sugerirle" que aquel proceso que encarnaban los huelguistas debería ser frenado, no por la policía o el ejército, sino por otras fuerzas sociales como lo eran los obreros y los campesinos. ${ }^{42} \mathrm{O}$ sea que el "jefe" ordenaba reprimir a los estudiantes contra ellos a las corporaciones para estatales obrero-campesinas, con el fin de resguardar el prestigio político del Estado. [...] En medio de esta violenta lucha ideológico-política de masas y de poderosos líderes reales, que se daba a la sombra del dejar hacer y dejar pasar del gobierno federal de Abelardo Rodríguez, fue que el general Calles, el Congreso local de Jalisco y el bloque de diputados federales revolucionarios propusieron que el Congreso de la Unión reformara el artículo tercero constitucional y eliminara la educación laica consagrada en su texto. A raiz de estas iniciativas fue que el Congreso designó una comisión para estudiar dicha solicitud. ${ }^{43}$

Demasiado pronto inició la violencia en la Universidad. En octubre de 1933 la mayoría de estudiantes estaba contra el rector Díaz de León, que como se ha dicho ya, al regresar del Congreso de Universidades, intentó aplicar sus acuerdos. Hubo estudiantes detenidos, parecía que se iba a iniciar una escalada represiva, presagiándose violencia de mayor grado, particularmente por la intervención del ejército y no solo de la policía, lo que muestra la importancia que el régimen otorgaba al conflicto. Agreguemos que los estudiantes liberados hicieron mofa de la represión con una manifestación festiva. ${ }^{44}$ Hubo otra represión el 17 de septiembre. La clausura de la Universidad

\footnotetext{
41 Ibid., 267.

42 Mendoza. Organizaciones y Movimientos Estudiantiles en Jalisco de 1900 A 1937 , 210-21, en: Rivera, el estudiantado, óp. cit., 53.

43 Rivera, el estudiantado, óp. cit., 52-53. Hasta aquí la transcripción seleccionada de Rivera.

44 Guevara, "Las luchas estudiantiles en México", 60-61.
} 
por Allende apaciguó los ánimos. La violencia no fue el tono de las jornadas de lucha para ninguno de los dos bandos, salvo los muertos el tres de marzo, de lo que se hablará más adelante.

Mientras tanto, las luchas se incrementan, se crispan los ánimos, algunos alumnos están armados, no por la amenaza de sus contrincantes estudiantiles sino por la actitud represiva de la policía. El 16 de noviembre la Normal de Mujeres es tomada por los huelguistas y no permiten intervenir a las fuerzas del orden, pues muestran sus armas desde la azotea del edificio. Se da un enfrentamiento y hay lesionados, entre estudiantes y policías, más de los primeros. Se les decomisan a los alumnos pistolas, navajas, puñales y parque. El gobernador responde al pliego petitorio de este grupo que exige autonomía y con ella libertad de cátedra. Pero, se encuentra en un dilema y argumenta el por qué no puede conceder esa propuesta. Explica que el gobierno si bien analiza todas las propuestas, su política debe estar acorde con las circunstancias de la época.

"La libertad de cátedra es aceptable solo en el sentido de que el Estado
no puede imponer dogmas y filosofías determinadas porque es conse-
cuente con el laicismo obligatorio -...] no puede permitir que al aparo
de la libertad puedan los enemigos de la emancipación revolucionaria de
México seguir atacando a las instituciones." 45

El movimiento estudiantil deja de ser un problema sectorial y se convierte en un asunto de todos los habitantes de la ciudad, los cuales ven atónitos cómo los estudiantes de uno y otro bando recorren todos los días las calles, toman escuelas, hacen mítines, reparten propaganda. La prensa es un reflejo de la toma de partido de la gente, de los voceros de empresas, de las instituciones civiles y eclesiásticas, de los editorialistas y de los propios medios de comunicación que hacen reportajes, entrevistas y notas periodísticas todos los días. ${ }^{46}$

Mientras la oposición estudiantil al "dogma marxista" se extiende por otras ciudades del país, demandando la autonomía universitaria y la libertad de cátedra, la corriente socialista es apoyada por múltiples organizaciones

\footnotetext{
45 Documento de la Secretaría General de Gobierno del Estado de Jalisco, 16 de Noviembre de 1933. AIP.

46 El periódico "El Jalisciense" se inclina a apoyar a los alumnos prosocialistas. El Informador, aunque no decididamente, por los autonomistas. Los corresponsales de Excélsior y el Universal también consignan en sus entregas los pormenores de los hechos, de manera más bien imparcial.
} 
locales y nacionales ${ }^{47}$ que se suman al Polo anti-huelguista estudiantil y magisterial. Por su parte, el 15 de Octubre la Asamblea del Consejo Directivo Nacional de la Confederación Nacional de Estudiantes (CNE), organización nacional poderosa y muy activa en la cual participan una delegación de Jalisco, acordó enfrentar la educación socialista universitaria por medio de la campaña pro-libertad de estudios, particularmente por la:

\begin{abstract}
[...] incongruente actitud de algunos gobiernos locales, exigiendo que los estudiantes universitarios nos mantengamos en pie de lucha para lograr de todos los gobiernos de los estados el respeto más absoluto para los centros de cultura del país, los cuales rechazan siempre todo intento de amordazamiento[...] La CNE se dirigirá a todas las universidades de América y de Europa para pedirles que se unan a la protesta de la juventud de México por la clausura de las Universidades de Monterrey, de Saltillo y Guadalajara. ${ }^{48}$
\end{abstract}

Sus baterías estaban enfocadas contra el comunismo, al que consideraban de suyo siniestro. Los mismos simpatizantes del socialismo cardenista se cuidaban de no ser identificados como comunistas.

En noviembre de 1934 en el Congreso de la Unión se aprobó la reforma del artículo tercero y con ello la versión de la educación socialista que concebían los diputados del PNR. ${ }^{49}$ El artículo en realidad no plateaba una versión socialista sino positivista, tal como lo señala Guevara Niebla, ${ }^{50}$-o "liberalpositivista" como refiere Rivera- ${ }^{51}$ al establecer que la educación se guiaría por una concepción exacta del hombre, del universo, de la vida social y acabar con todos los prejuicios. Para los simpatizantes del reformismo del Estado veían claramente esta acepción como coherentemente socialista, la cual estaba sin duda basada en la versión dogmática que planteaba entonces el materialismo dialéctico. De ahí que el Partido comunista con una formación más profunda de las teorías socialistas no haya apoyado en un principio la reforma socialista del Estado.

\footnotetext{
47 El PNR, el Partido Estudiantil Revolucionario Cardenista y el Comité estudiantil Pro-Everardo Topete la Cámara del trabajo, la gran Logia de Occidente, el Grupo Vanguardia 1910, el Partido liberal Obrero de Jalisco, el Comité pro-industria, la Liga de Electricistas jaliscienses, el grupo Anticlerical, el Círculo feminista de Occidente, el Centro de Orientación y Pedagógico de Maestros y la nueva directiva de Estudiantes Normalistas. Romero, Jalisco Desde La Revolución. Tomo 5: Movimientos Sociales. 1929-1940. Gobierno Del Estado De Jalisco: Universidad De Guadalajara, 1988., 282, en: Rivera, el estudiantado, óp. cit., 58.

48 Periódico Excélsior, en: Guevara, "Las luchas estudiantiles en México", 83-84.

49 Según Rivera, poco tiempo después se adicionó la Ley Reglamentaria correspondiente, pero esa reglamentación nunca se haría, hasta que volvió a reformarse dicho artículo con Ávila Camacho y se excluyó el sentido socialista de la educación.

50 Guevara Niebla, Gilberto. Saber y Poder, (México: Universidad autónoma de Sinaloa, 1983).

51 "Y que ya examinados con rigor seguían siendo puramente liberales." Rivera, el estudiantado, óp. cit., 61.
} 
El Frente de Estudiantes Socialistas de Occidente fue la organización más integrada, más madura y tenaz de todas los que surgieron en los años treinta. Toda la concepción sobre el tipo de educación que debía establecerse, qué ideología sostener y que formas de ejercicio político debía organizarse, se proyectaron con un haz de luz potente en quienes organizarían la universidad durante al menos tres décadas. Su raíz más evidente es el vínculo con el estado cardenista, pero había que agregar a ese antecedente la generación que se nucleó en su entorno. Esta también, como antecedente simbólico pero no reconocido, la influencia de los estudiantes comunistas en la Universidad que estuvieron difundiendo las teorías marxistas entre el estudiantado de las escuelas superiores a los pocos años de la fundación del partido comunista en 1919: "Los estudiantes comunistas hicieron acto de presencia en la Universidad de Guadalajara desde 1931 en el comité pro-unificación Normalista de Guadalajara y en los círculos universitarios marxistas, cuya existencia fue decisiva para lo que vino después. Se trataba de un proceso en parte espontaneo pero también inducido por el Estado mexicano". ${ }^{52}$

Las ideas que exhalaban la lucha contra las fuerzas oscuras y retrógradas y los planteamientos de conquistar un mundo de justicia social fueron calando en los sectores que habían mantenido neutrales y al margen del conflicto, y porque vieron que esa bandera constituía una política que se publicitaba desde el Estado mismo. ${ }^{53}$ En este sentido, la Universidad de Guadalajara será arena donde habrán de socializarse estas ideas que pregonaban la educación popular, la lucha por hacer factible el cambio social pregonado por la revolución, luchar contra los grupos reaccionarios y religiosos. He ahí el caldo de cultivo para un sector de alumnos universitarios (entre ellos los normalistas, pues las Escuelas Normales estaban integradas a la Universidad).

La fundación del FESO es la muestra más palpable de que los sectores "revolucionarios" tenían más sentido de lo que quería y cómo iban a lograrlo, pero las organizaciones contrarias tenían mayoría porque aprovecharon la poca formación política del estudiantado, adhiriéndolos más que nada por intuición y exaltación y desde luego creando en ellos alarma y miedo, advirtiendo lo fatal de las ideas perversas y comunistas que traban de imponer el bando contrario.

\footnotetext{
52 Rivera, el estudiantado, óp. cit., 61.

53 Puede evidenciarse en los desplegados de adherencia de diversas agrupaciones sociales y políticas al FESO y los socialistas, así como en la correspondencia que publican los periódicos referentes al problema. Todo ello indica el crecimiento de esa tendencia entre la población de Jalisco. Organizaciones y Movimientos op. Cit.
} 


\section{CONCLUSIÓN}

El apoyo del gobierno del Estado tenía que derivar en la constitución de un modelo universitario propio para Guadalajara, muy cercano a los propósitos del gobierno cardenista. De esta manera, la Universidad de Guadalajara fue refundada en 1937 bajo lineamientos propios y en donde el grupo que defendió y luchó por esta propuestas y que no era otro que el grupo que encabezaba Enrique Díaz de León, se responsabilizó de la configuración de su modelo y su operación, recibiendo desde entonces el apoyo financiero y político del Estado convirtiéndose en la institución universitaria oficial de Jalisco. El vínculo entre el gobierno Federal y el FESO fue fundamental. Hoy podríamos preguntarnos si esta organización en realidad no fue concebida y organizada por el propio estado cardenista (o discrecionalmente incluso por el gobierno del Estado en turno) para tener una universidad adherida a sus interese educativos e ideológicos. O por el contrario, tuvo el FESO una concreción propia y autogestiva y que el apoyo que recibió fue fundamentalmente por el vínculo coincidente entre ambas instancias. Es sintomático que gran parte de sus reuniones se realizaban regularmente en las propias instalaciones de la Universidad y recibiendo desde su fundación el auspicio del Estado, traducido en recursos económicos y el otorgamiento de todas sus peticiones, cabildeo permanente ante el gobierno Estatal, incluso con el propio presidente de la República para enfrentar o desterrar a los grupos opositores, siendo todo ello evidente, así como la utilización de los cuerpos policiacos como instrumento para reprimir a sus enemigos.

Por su parte, los miembros del conglomerado autonomista, no fueron finalmente consecuentes con sus postulados de libertad de cátedra ni la autonomía, pues al final se rindieron rápidamente ante la propuesta de que podrían fundar una institución privada y hacia allí enfocaron todas sus baterías, dejando regadas por el camino todas las convicciones que habían publicitado y defendido, puesto que aceptar refugiarse en una visión restringida de la misión educativa (por su carácter privado minoritario), echaba a la basura su alegato autonomista, pues ¿qué importa la autonomía en una institución que se regirá por un régimen privado? Asimismo, ¿dónde queda la libertad de cátedra? Pues al regirse la universidad por los cánones de una mentalidad y racionalidad directiva y empresarial cómo demanda una institución privada, ¿podría sostener su concepto de libertad de cátedra? No fue así, por 
lo que detrás de la retórica académica emancipadora y su lucha contra la doctrina socialista fue orientándose hacia la ultraderecha.

\section{FUENTES}

Periódicos: El Informador, (Guadalajara, Jalisco, 1933-1937, notas periodísticas sin autor). El Jalisciense, (Guadalajara, Jalisco, 1933-1938, notas periodísticas sin autor). El Universal, (Cd. De México, 1933-1937, de su corresponsalía en Guadalajara, Jalisco) El Machete, órgano oficial del periódico del Partido Comunista Mexicano (México D.F. notas de su corresponsalía en Guadalajara, Jalisco). Las fechas de las notas periodísticas se incluyen en las referencias a pie de página.

\section{REFERENCIAS}

Campbell, Hugh Gerard. La Derecha Radical En México, 1929-1940. México: Colección Sepsetentas, 1976.

Dorantes, Alma. El Conflicto Universitario En Guadalajara 1933-1937. Guadalajara: INAH/SC, 1993.

García Diego, Javier. "El Proyecto Universitario de Justo Sierra: Circunstancias y Limitaciones". En Cultura y Política en el México Posrevolucionario México: INEHRM, 2006.

Guevara Niebla, Gilberto. Las Luchas Estudiantiles En México. Tomo II. México: UAG/ AUZ, 1986.

Guevara Niebla, Gilberto. Saber y Poder. México: Universidad Autónoma de Sinaloa, 1983.

Mantilla Trolle, Marina y Armando Martínez Moya. “Jalisco a la hora de su Universidad". En Universidad de Guadalajara: más de dos siglos de Historia Editado por Guadalajara: Universidad de Guadalajara, 2013.

Martínez Moya, Armando. "Voces de Huelga desde El Corredor". En Universidad de Guadalajara: Más de dos Siglos de Historia. Guadalajara: Universidad de Guadalajara, 2013.

Mendoza Cornejo, Alfredo. Antología Del Pensamiento de Enrique Díaz De León. Guadalajara: Colección Universitarios Ilustres. Vol. III. U.D.G., 1988.

Mendoza Cornejo. Alfredo Enrique Díaz de León. “Discurso de Inauguración del Congreso de Universitarios Mexicanos". Guadalajara: Testimonios Universitarios, 1983.

Mendoza Cornejo, Alfredo. Organizaciones y Movimientos Estudiantiles en Jalisco de 1900 A 1937. Guadalajara: Editorial Universidad de Guadalajara, 1989. 
Mendoza Cornejo, Alfredo. Enrique Díaz De León. Político, Ideólogo y Pedagogo de la Universidad de Guadalajara. . Guadalajara: Editorial Universidad de Guadalajara,1992.

Rivera Ortiz, Mario. El Estudiantado Una Nueva Clase Social. S/E. México: Guadalajara, 2007.

Romero, Laura Patricia (Coordinadora). Jalisco Desde La Revolución. Tomo 5: Movimientos Sociales. 1929-1940. Gobierno Del Estado De Jalisco: Universidad De Guadalajara, 1988.

Rovira, María del Carmen. "El Ateneo de la Juventud". En Una Aproximación a la Historia de las ideas Filosóficas En México. Siglo XIX y principios del XX . México: UNAM, 1997.

Rumbo A La Universidad. Testimonio De La Polémica Antonio Caso/Lombardo Toledano. Colección Metro. No 1.- México 1973.

Valles, Patricia. “La Derecha Jalisciense en el Periodo de la Consolidación del Estado Mexicano (1929-1940)". En Miscelánea Jalisciense. Guadalajara. Colegio de Jalisco, 1999. Zuno, José Guadalupe. "Reminiscencias de una vida". Guadalajara: Universidad de Guadalajara, 2011.

Martínez Moya, Armando. "Libertad de cátedra o socialismo de Estado. El dilema de los estudiantes de la Universidad de Guadalajara en 19331937”. Revista Historia de la Educación Latinoamericana. Vol. 16 No, 22, (2014): $191-211$. 